\title{
Middleware-Controlled Resource consumption for Location Traffic in Cellular Networks
}

\author{
Israel Martin-Escalona and Francisco Barcelo
}

\begin{abstract}
Location is valuable information for services implemented in wireless networks. Location systems often use the infrastructure of cellular networks that have already been deployed. Accordingly, location systems spend resources of the network they use. This paper proposes a middleware to reduce the consumption of network resources and optimize the location traffic that is being carried. This middleware, called MILCO (Middleware for Location Cost Optimization), selects the optimum location technique depending on the request, i.e. the location technique that satisfies the quality of service (QoS) required and minimizes the resource operating expense. In addition, MILCO takes advantage of ongoing and carried location processes to reduce the overall expenditure of resources. Our results show that MILCO can reduce location-process failures and improve latency figures for location provisioning and resource use in cellular networks such as UMTS.
\end{abstract}

Index terms: LBS, middleware, resource-consumption optimization, QoS, UMTS.

\section{INTRODUCTION}

Mobility is a key factor in the provision of services in cellular networks. Although most of the technical limitations (error rate, bit rate, etc.) of $2 \mathrm{G}$ and $2.5 \mathrm{G}$ networks have been overcome in $3 \mathrm{G}$ networks, services do not seem to have taken off. Most experts agree that in order to revitalize the services market, new wireless services that offer real added value must be developed. This does not just mean transferring services from the wired to the wireless context, but also developing new value-added services for wireless environments. Location based services (LBS) are an example of these new services.

Many operators regard location as a feature for advanced services on wireless networks that will become crucial in the near future. Location is a standalone service (i.e. the user wishes to know his/her position) and, at the same time, a lower layer for other services and applications (e.g. the user does not need to know his/her position, but the service requested needs the position to be provided). In addition, the key role of location services (LCSs) for public safety and emergency purposes has led regulators to increase quality requirements. Location is also useful to operators further than the revenue

Manuscript received July 07, 2006 and revised November 20, 2006. This research was partially funded by the EC through the 6th FP IST Liaison project and by the ERDF and the Spanish government through project TIC2003-01748. This paper was presented as part at the Next Generation Teletraffic and Wired/Wireless Advanced Networking (NEW2AN) 2006.

I. Martin-Escalona and F. Barcelo are with the Technical University of Catalonia (UPC), c/ Jordi Girona 1-3, Edifici C3, Barcelona, Spain (e-mail: \{imartin, barcelo\}@entel.upc.edu) they may obtain from providing it. This is because location information can be used to optimize the management of network resources [21, 22, 23], by taking advantage of, for example, intelligent paging and intelligent resource allocation for handoff $[24,25]$ [in the case of the latter, the network knows the location, speed and direction of the mobile station (MS) and can therefore reserve a channel for handoff in the next cell].

So far, only a few LBS have been implemented for the mass market, although the recent deployment of the latest generation of $3 \mathrm{G}$ networks has removed some of the factors delaying their introduction, such as low-bandwidth channels and the lack of definition of the location system architecture and protocol stacks. However, other issues remain, the most significant of which is probably the mismatch between the quality of service (QoS) offered by location techniques and the QoS required by LBS.

Table I shows the QoS required by the main location based services [20], where Acc stands for the expected accuracy of the service.

TABLE I

QoS OF THE MAIN LOCATON BASED SERVICES

\begin{tabular}{|lrrrrrr|}
\cline { 2 - 7 } \multicolumn{1}{c}{} & \multicolumn{2}{c}{ Indoor } & \multicolumn{2}{c|}{ Urban } & \multicolumn{2}{c|}{ Suburban } \\
\hline LBS & Acc & Yield & Acc & Yield & Acc & Yield \\
\hline $\begin{array}{l}\text { Information services } \\
\text { Basic } \\
\text { Enhanced }\end{array}$ & $\begin{array}{r}50-100 \mathrm{~m} \\
20-50 \mathrm{~m}\end{array}$ & $\begin{array}{r}80.0 \% \\
90.0 \%\end{array}$ & $\begin{array}{r}50 \mathrm{~m} \\
50 \mathrm{~m}\end{array}$ & $\begin{array}{r}80.0 \% \\
90.0 \%\end{array}$ & $\begin{array}{r}1000 \mathrm{~m} \\
50 \mathrm{~m}\end{array}$ & $\begin{array}{r}80.0 \% \\
90.0 \%\end{array}$ \\
\hline Navigation & $10 \mathrm{~m}$ & $95.0 \%$ & $10 \mathrm{~m}$ & $99.9 \%$ & $10 \mathrm{~m}$ & $99.9 \%$ \\
\hline $\begin{array}{l}\text { Worker } \\
\text { management }\end{array}$ & $50 \mathrm{~m}$ & $95.0 \%$ & $50 \mathrm{~m}$ & $95.0 \%$ & $50 \mathrm{~m}$ & $95.0 \%$ \\
\hline $\begin{array}{l}\text { Worker } \\
\text { tracking }\end{array}$ & $50 \mathrm{~m}$ & $99.9 \%$ & $50 \mathrm{~m}$ & $99.9 \%$ & $50 \mathrm{~m}$ & $99.9 \%$ \\
\hline $\begin{array}{l}\text { On-demand } \\
\text { transport }\end{array}$ & $\mathrm{n} / \mathrm{a}$ & $\mathrm{n} / \mathrm{a}$ & $50 \mathrm{~m}$ & $90.0 \%$ & $50 \mathrm{~m}$ & $90.0 \%$ \\
\hline $\begin{array}{l}\text { Children } \\
\text { tracking }\end{array}$ & $50 \mathrm{~m}$ & $99.9 \%$ & $50 \mathrm{~m}$ & $99.9 \%$ & $\begin{array}{r}50- \\
100 \mathrm{~m}\end{array}$ & $99.5 \%$ \\
\hline $\begin{array}{l}\text { Medical } \\
\text { alert }\end{array}$ & $50 \mathrm{~m}$ & $99.9 \%$ & $50 \mathrm{~m}$ & $99.9 \%$ & $\begin{array}{r}50- \\
100 \mathrm{~m}\end{array}$ & $99.9 \%$ \\
\hline
\end{tabular}

At present, several location techniques are ready for deployment: cell identification, terrestrial signal trilateration, satellite navigation, finger-printing, angle of arrival, etc. Each of them provides a given quality of service that is usually measured in terms of accuracy, response time, availability and consistency [1], the first two of which are considered to be the most relevant. There are a wide variety of LBS; each, however, requires a different QoS depending on the purpose of the service. Thus, the capabilities of location systems for carrying location requests from different LBS depends on the features of the location techniques implemented in them [2]. 
Table II shows the QoS obtained by the most popular location techniques. This table shows that none of the location techniques perform to a high standard under all of the possible conditions. For example, the availability of cell identification methods may be very good but accuracy and consistency are usually poor since they depend on cell size; GPS techniques, on the other hand, give accurate positions but availability is poor indoors, etc.

TABLE II

QoS of the MaIn Location techniques

\begin{tabular}{|l|l|l|l|l|}
\hline Technique & Accuracy & $\begin{array}{c}\text { Response } \\
\text { time }\end{array}$ & Availability & Consistency \\
\hline Cell ID & Fair/Poor & Very good & Very good & Very poor \\
\hline $\begin{array}{l}\text { Signal } \\
\text { strength }\end{array}$ & Poor & Good & Very good & Very poor \\
\hline TOA/TDOA & Good & Good & Fair & Good \\
\hline AoA/DoA & Good & Good & Fair/Poor & Fair \\
\hline Fingerprint & Good & Fair & Good & Fair \\
\hline GPS & Very good & Fair & Good & Good \\
\hline $\begin{array}{l}\text { Hybrid } \\
\text { systems }\end{array}$ & Very good & Poor & Very good & Good \\
\hline
\end{tabular}

Hybrid techniques are proposed to overcome the shortcomings of using location techniques as standalone. They are based on combining measurements taken using a variety of techniques in order to take advantage of the particular strengths of each technique [3-7]. The use of different techniques enhances the QoS offered by the system and allows more LBS to be carried. However, the QoS figures obtained using different techniques are often much higher than necessary for many LBS, which can lead to an inefficient use of network resources.

This paper proposes a new approach to optimizing the use of resources in location systems. It is organized as follows. The approach proposed is explained in Section 2. Section 3 presents the simulation tool used to carry out the analysis and the scenarios being simulated, while Section 4 analyzes the results obtained. Finally, in Section 5 the main conclusions are summarized.

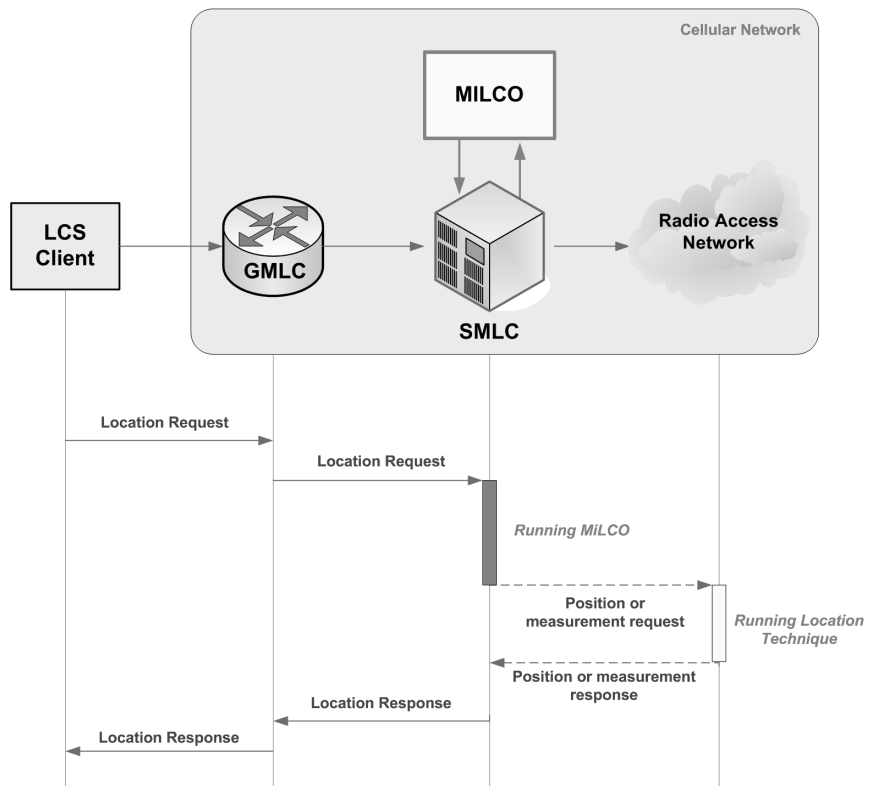

Fig. 1. Location system integrating MILCO

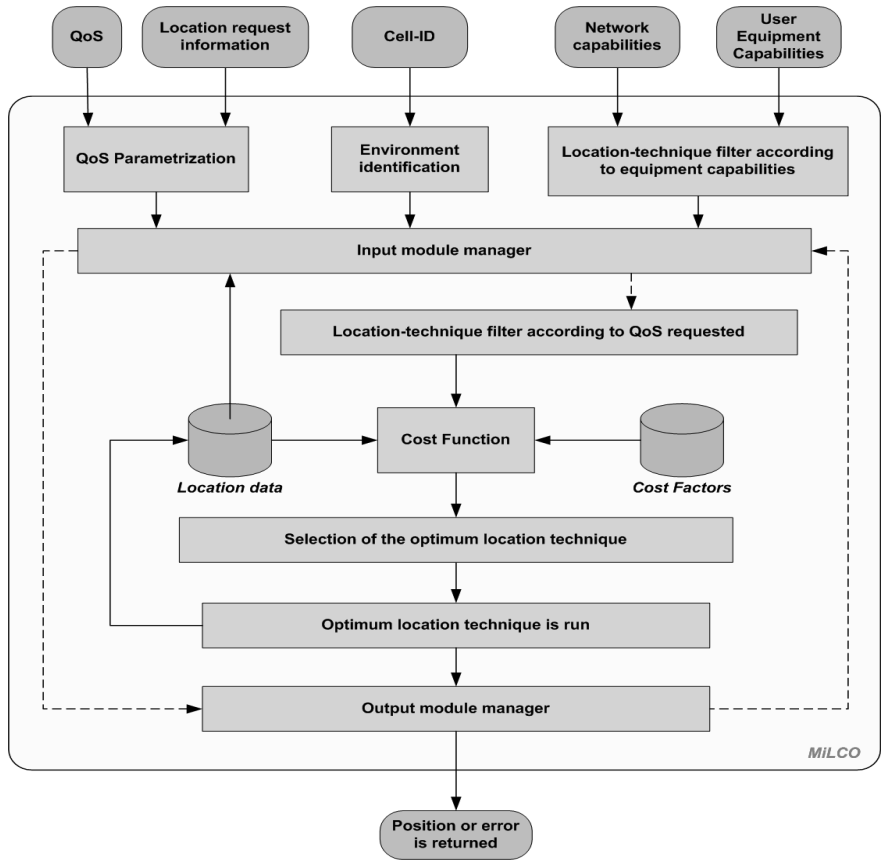

Fig. 2. Block diagram of MILCO

\section{MILCO}

\section{A. System definition}

The network resources consumed by the location system belong to the infrastructure of the underlying cellular network on which the location service is running. Consequently, the resources used for location purposes are not available to other traffic. This paper proposes using a middleware to optimize the use of resources in location systems: MILCO (Middleware for Location Cost Optimization) [8]. MILCO manages all location processes and is aimed at reducing resource use as long as the QoS requested is fulfilled. There are several proposals for location middlewares, but they are focused on technology independence, system integration and rapid development of LBS [9-11], rather than on the efficient use of resources.

MILCO is implemented as a new piece of software in the location managers, e.g. in the Serving Mobile Location Centers (SMLCs) in the case of ETSI/3GPP notation [12]. Fig. 1 shows a location system architecture that includes MILCO. ETSI/3GPP notation has been used for reference purposes. Every time a location request reaches the location system via the GMLC, it is delivered to the MILCO entity. MILCO then selects the location technique that best fits the request, i.e. that which is expected to achieve the requested QoS and which minimizes the use of resources. Finally, MILCO uses the network's facilities to ascertain the user's position and forward the result to the Location Service (LCS) client that requested it.

Fig. 2 shows the steps that define MILCO's performance for each location request: filtering, location-technique selection and result management. The filtering stage involves all the blocks above the cost function and is aimed at filtering out any location technique that is not appropriate to the request. 
Location techniques may be marked as unsuitable for three reasons: incompatibility (i.e. either the network or the user terminal is unable to implement the technique); the location technique is unable to achieve the QoS requested (e.g. the maximum accuracy achieved by the technique is worse than that requested); or an input module is able to handle the request without running the location technique. The second stage is location-technique selection. During this stage, MILCO selects the optimum location technique from the remaining set (i.e. after filtering). This is achieved by means of a cost function, which ranks the resource consumption of all the location techniques. Finally, the third stage manages the results, i.e. it chooses the procedures that will be used to handle failures and maintain a database with the previous location measurements and calculations, etc. The default behavior in the event of location failure is to execute another location technique. Note that, in these cases, the requirement for the response time may be much more constrained, since time has already been spent on previous location attempts.

\section{B. Cost function}

The cost function is MILCO's core module. It ranks the location techniques that are suitable for the request according to their use of resources, i.e the more resources the technique uses, the lower it is ranked. This rank is further used to select the optimum location technique, i.e. the one that uses the fewest resources.

The cost function is composed of several factors, which are used to quantify network resource use. Thus, it is defined as

$$
Z\left(L T_{i}, t\right)=f\left(\alpha_{1}, \ldots, \alpha_{n} ; z_{1}\left(L T_{i}\right), \ldots ., z_{n}\left(L T_{i}\right) ; t\right),
$$

where $Z(L T i)$ represents the resources spent by the $i^{\text {th }}$ location technique (i.e. $\left.L T_{i}\right), f$ stands for a given function, $\alpha_{j}$ and $z_{j}\left(L T_{i}\right)$ are, respectively, the weight and value of the $j^{\text {th }}$ factor applied to location technique $L T_{i}$ and $t$ is the time at which the resource-consumption is going to be calculated. Several functions $(f)$ can be used to calculate resource use. This paper proposes using a simple additive function with $m$ factors to evaluate the performance of the module. It is defined as

$$
Z\left(L T_{i}, t\right)=\sum_{i=1}^{m} \alpha_{i} \cdot z_{i}\left(L T_{i}, t\right)
$$

The factors taken into account in this paper and whose use is penalized are the signaling volume, the use of lowbandwidth channels and energy consumption at the user terminal [8]. More details on these parameters are provided in the following sections.

\section{B.1 Signaling volume}

The signaling volume penalizes techniques that involve exchanging large amounts of data. In the computation of the signaling volume only the topmost protocol in the stack was taken into account, i.e. the RRC [26] for all techniques except Cell-ID, for which all information is supplied in the location request. The RRC involves transmission of two location messages. First of all, a measurement control message is sent from the radio access network to the mobile station to request the execution of a location technique and provide any additional data that is necessary. If the execution is successful, a measurement report message is then sent back to the radio access network, including the measurements requested or the position computed. Details of the specific fields used for location in this research are given in Tables III and IV, where $M P$ indicates mandatory, $O P$ stands for optional, $\mathrm{CH}$ refers to choice and $C V$ indicates that the presence of the information element (IE) may be mandatory or optional depending on the values of other Ies.

TABLE III

\begin{tabular}{|c|c|c|}
\hline Information element & Presence & Length (bits) \\
\hline Message type & MP & 6 \\
\hline UE information elements & & -- \\
\hline RRC transaction identifier & MP & 4 \\
\hline Integrity check info & $\mathrm{CH}$ & 36 \\
\hline Measurement information elements & & -- \\
\hline Measurement identity & MP & 4 \\
\hline Measurement command & MP & 2 \\
\hline Measurement reporting mode & $\mathrm{OP}$ & 2 \\
\hline Additional measurements list & OP & $4 . .16$ \\
\hline UE positioning measurement & $\mathrm{CV}$ & \\
\hline \multicolumn{3}{|c|}{$\begin{array}{r}\text { OTODA }: 35 \\
\text { OTODA (needing assistance data) }: 142+N_{B S} * 134 \\
A+G P S: 391+N_{S A T} * 647 \\
A+G P S \text { (including almanac): } 403+\mathrm{N}_{\mathrm{SAT}} * 1199 \\
\text { Indoor A+GPS: } 476+\mathrm{N}_{\mathrm{SAT}} * 702 \\
\text { Indoor A+GPS (including almanac): } 488+\mathrm{N}_{\mathrm{SAT}} * 1254 \\
\text { Hybrid (needing assistance data): } 583+N_{B S} * 134+\mathrm{N}_{\mathrm{SAT}} * 1254\end{array}$} \\
\hline \multicolumn{3}{|c|}{\begin{tabular}{|l|l|l|} 
Physical channel information elements & & \\
\end{tabular}} \\
\hline DPCH compressed mode status info & $\mathrm{OP}$ & -- \\
\hline
\end{tabular}

RRC Measurement Control message

\section{B.2 Low-bandwidth channel use}

The use of low-bandwidth channels favors techniques that use wide-band channels. Accordingly, the location techniques that are most severely penalized by this factor are those that cross the radio access network, where the most restrictive interfaces are placed. This usually applies to the most accurate location techniques (e.g. A-GPS). Other techniques, such as Cell-ID or RTT, use core-network facilities to compute the position, at the expense of accuracy [27]. The bandwidth use is computed in this work as

$$
z_{c u}\left(L T_{i}\right)=\sum_{i=1}^{N_{\text {inerfice }}}\left[\operatorname{Thr}\left(I_{i}\right)\right]^{-1},
$$

where $z_{c u}$ indicates the cost factor result, $N_{\text {interface }}$ the number of interfaces crossed by the location technique $L T_{i}$ and $\operatorname{Thr}\left(I_{i}\right)$ the throughput of the $i^{\text {th }}$ interface. This cost factor does not account for the amount of data sent through each of the interfaces; it only accounts for the capabilities of these interfaces.

\section{B.3 Energy consumption}

The aim of this cost factor is to penalize techniques that quickly deplete the user's terminal battery. The amount of energy consumed by each location technique mainly depends on the hardware used and the software that uses this hardware. Hence, it is very difficult to rank the location techniques' energy consumption. Therefore, the authors decided to compute this cost factor according to the number of signals that the user equipment needs to track to carry out the location. It is assumed that tracking of radio signals is a hard-optimized 
task in all the technologies, so the techniques can be compared.

TABLE IV

RRC Measurement Report message

\begin{tabular}{|l|c|r|}
\hline Information element & Presence & Length (bits) \\
\hline Message type & MP & 6 \\
\hline UE information elements & & -- \\
\hline Integrity check information & CH & 36 \\
\hline $\begin{array}{l}\text { Measurement information } \\
\text { elements }\end{array}$ & & -- \\
\hline Measurement identity & MP & 4 \\
\hline Measured results & OP & 117 \\
\hline Measured results on RACH & OP & -- \\
\hline Additional measured results & OP & -- \\
\hline$>$ Measured results & MP & -- \\
\hline Event results & OP & -- \\
\hline GSM OTD reference cell & OP & -- \\
\hline
\end{tabular}

\section{Input modules}

Input modules are used to extend the functionalities of the cost function and improve its performance. There are two input modules in the design: the location cache and the concurrence manager. The location cache prevents having to run a location technique whenever the user position can be estimated with a reasonable degree of accuracy. The location cache works on the basis of two hypotheses: that an older position is available to the user and that the user is close to this position. There are several approaches to verifying whether the terminal position is close enough to the last stored position [13]. MILCO builds a database using the results of previous location processes and uses the age of the stored positions as a constraint on the location cache, i.e. it only uses old stored positions when they are not older than a given threshold value. If this is the case, the average speed of the terminal (calculated from the data stored in the database) is used to estimate the current position of the mobile station. Thus, depending on the QoS required, this estimation may be sufficient and may help to avoid using new resources. Note that the more static the users are, the better the expected performance.

Concurrence aims to avoid collisions in the execution of location techniques. A collision happens whenever a location request for a specific user is received while another one, for which higher QoS has been requested, is in progress. In such situations, the concurrence manager blocks the last request received until the ongoing one ends. The resulting position is then shared by the two requests, even though the QoS returned for some of them is better than required. This procedure should become more effective as the location traffic (per user) increases.

The location cache and concurrence manager reduce the number of requests that reach the cost function; hence, no location techniques are run for requests that use these modules. As a result, the overall amount of resources used for location is also reduced.

\section{SimULATOR AND SCENARIOS}

\section{A. Network parameters}

The simulator used in this paper was developed specifically to test MILCO. It allows any kind of network and cellular scenario to be simulated (e.g. channel allocation method, mobility and propagation patterns, admission and power control algorithms, etc.). The simulator double-wraps the simulation area to minimize the impact of the edge effect on the results. This means that the simulation area becomes a torus [14], i.e a virtually infinite surface from mobility and propagation points of view.

This paper evaluates MILCO on urban cellular UMTS networks. Admission control [15] and power control [16] algorithms were implemented. The control algorithm accepted new users whenever the target SIR of any of the ongoing calls in the cell did not drop by more than $1 \mathrm{~dB}$. The power control parameters are given in Table $\mathrm{V}$. We proposed simulating a basic scenario in which several location loads ranging from 1 request every 30 seconds to 2 requests per second were applied. Fig. 3 shows the resulting layout, which was composed of 100 Node Bs (NB) uniformly distributed across the simulation area. Each Node B was placed in the center of a square-shaped building and achieved a cell coverage of 1135 meters. In this case, a significant proportion of the whole area was subject to overlapping, i.e. was covered by more than one Node B. This meant that the simulation was closer to reality; it also allowed OTDOA, which is not possible in areas covered by just one or two Node Bs.

TABLE V

Parameters of the POWER CONTROL ALGORithm

\begin{tabular}{|l|r|}
\hline Parameter & \multicolumn{1}{|c|}{ Value } \\
\hline$\eta_{0}=\eta_{1}=\eta_{01}$ & 2 \\
\hline$\Delta_{0}=\Delta_{1}=\Delta_{01}$ & 10 \\
\hline$\Delta_{\max }$ & $10 \mathrm{~dB}$ \\
\hline$\Delta_{\min }$ & $0.01 \mathrm{~dB}$ \\
\hline$\Delta_{\text {initial }}$ & $1 \mathrm{~dB}$ \\
\hline Power updates between consecutive movements & 20 \\
\hline
\end{tabular}

Users moved freely within the simulation area and were able to enter these buildings. As the buildings are indoor areas, signal reception inside them was limited. The scenario was populated by a single pedestrian user. More users were not needed in this preliminary evaluation, since the performance of MILCO is user-oriented. MILCO takes decisions according to the location request's features, whose ultimate target is a specific mobile station. The inclusion of a number of mobile stations constrains the access network (e.g. mobile-based location techniques, the power control algorithm, etc.); its effect on MILCO will be the subject of further research. The user speed (in both directions, $x$ and $y$ ) followed a normal random variable, with mean and standard deviation of 0.59 $\mathrm{m} / \mathrm{s}$ and $0.17 \mathrm{~m} / \mathrm{s}$, respectively. The value of the user speed in both directions was updated once per second.

The propagation pattern followed the Okumura-Hata model, with path-loss slope and zero-meter losses set to 4 and $23 \mathrm{~dB}$, [17] respectively. The SIR was calculated in accordance with[18]; the spreading and orthogonality factors were $10 \mathrm{~dB}$ and 0.4 respectively [17]. Handoffs were requested every time the received power or the SIR in a Node B or mobile station (MS) fell below a threshold value (known as the handoff threshold). The handoff request was kept on hold until a new channel became free, as long as the received power or the SIR was between the handoff threshold value and the level of 
sensitivity of the terminal. If the SIR or received power fell below the sensitivity level, handoff was attempted for 15 seconds at the most. If a channel did not become free during this time, the service was interrupted and the user terminal backed off for an exponential time of 5-second mean. Note that successful handoffs drop all ongoing requests carried by the mobile station. Table VI shows the main parameters of the propagation pattern, which were extracted from $[17,18]$.

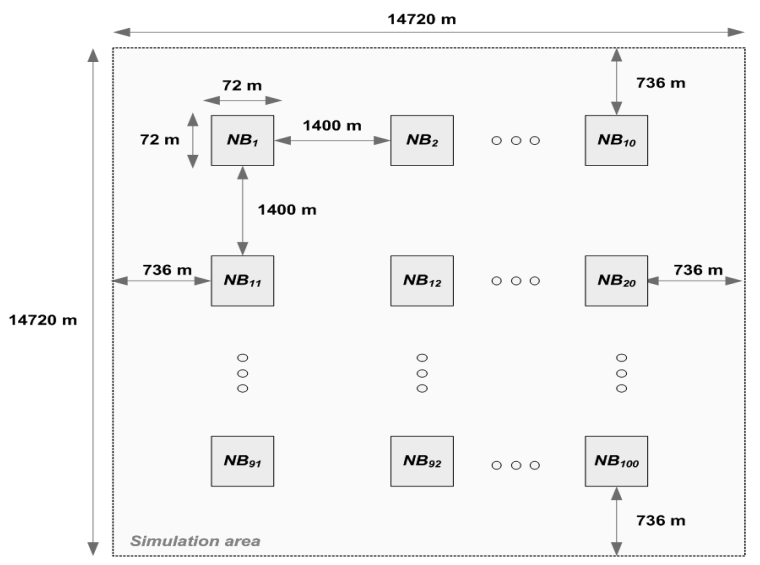

Fig. 3. Layout of the scenarios simulated

\section{B. Location-related parameters}

All the location techniques that are available in UMTS were taken into account in this scenario (i.e. Cell-ID, OTDOA and A-GPS). A hybrid, tightsynchronized OTDOA/A-GPS location technique [3] was also included to show the features of the hybridization upgrade. Table VII shows the accuracy and response times achieved by each of them[19]. In this table, mean is the average value, range indicates the set of values that the variable can take, std is the standard deviation, det stands for deterministic and n.a. means that the parameter does not apply. The availability of the OTDOA was computed according to the received power and the SIR. Three or more BSs must be visible in the MS for the OTDOA to be run. Otherwise, the OTDOA is considered to be unavailable at that time and position. In the case of satellite-based techniques, availability was checked differently. The scenario defined the default number of satellites in sight as 5. However, in indoor areas this figure was uniformly distributed from 1 to 2 satellites.

\section{TABLE VI}

PARAMETERS OF THE PROPAGATION PATTERN

\begin{tabular}{|l|r|}
\hline Parameter & Value \\
\hline Minimum SIR & $-9 \mathrm{~dB}$ \\
\hline Sensitivity of the stations & $-109.2 \mathrm{dBm}$ \\
\hline Maximum MS transmission power & $21 \mathrm{dBm}$ \\
\hline Minimum MS transmission power & $-44 \mathrm{dBm}$ \\
\hline Node B transmission power & $43 \mathrm{dBm}$ \\
\hline Handoff threshold for received power & $-106.2 \mathrm{dBm}$ \\
\hline Handoff threshold for SIR at reception & $-6 \mathrm{~dB}$ \\
\hline
\end{tabular}

All the features of MILCO, that is, the cost function and input modules, were implemented. Positions remained cached for 2 seconds. The cost function used in the scenarios is presented in (2). The quantification of the cost factors is shown in Table VIII, where $N_{N B}$ and $N_{S A T}$ are the number of Node Bs and satellites involved in the positioning. Two modes are specified in Table VIII for OTDOA and A-GPS: assisted (AS) and non-assisted (NAS). The assisted mode involves sending the assistance data to the mobile station, while the non-assisted mode assumes that this information has already been sent. Simulations were carried out under the assumption that the assistance data (for OTDOA and A-GPS) expires after 30 seconds, i.e. new assistance information is required 30 seconds after it has been received. Low-bandwidth channel use was quantified by counting the number of times an interface was crossed and dividing this figure by the throughput of the channel used. Energy consumption heavily depends on user terminal performance. Therefore, the authors proposed quantifying the energy consumption according to the number of signals transmitters involved in the location technique.

TABLe VII

QoS ACHIEVED By the LOCATION TECHNIQUeS

\begin{tabular}{|c|l|r|r|r|r|}
\cline { 3 - 6 } \multicolumn{2}{c|}{} & \multicolumn{5}{c|}{ Location techniques } \\
\cline { 3 - 7 } \multicolumn{2}{c|}{} & Cell ID & \multicolumn{1}{c|}{ OTDOA } & \multicolumn{1}{c|}{ A-GPS } & \multicolumn{1}{c|}{ Hybrid } \\
\hline \multirow{3}{*}{ Accuracy } & Distribution & Det. & Uniform & Gaussian & Gaussian \\
\cline { 2 - 7 } & Range $(\boldsymbol{m})$ & n.a. & {$[50 . .250]$} & n.a. & n.a. \\
\cline { 2 - 7 } & Mean $(\boldsymbol{m})$ & 1135 & 150 & 3 & 50 \\
\cline { 2 - 7 } & Std $(\boldsymbol{m})$ & 0 & 57.73 & 0.90 & 15 \\
\hline $\begin{array}{c}\text { Response } \\
\text { time }\end{array}$ & Distribution & Det. & Exponential & Exponential & Exponential \\
\cline { 2 - 6 } & Mean (s) & 0 & 7 & 11 & 27 \\
\hline
\end{tabular}

The weight of the factors in the cost function was the same and the maximum value of a weighted factor was set to 1 . Thus, the maximum value of the cost function was 3 . The weights $\left(\alpha_{j}\right)$ applied to the factors in order to achieve this behavior were $1.3651 \cdot 10^{-4}, 1.9152 \cdot 10^{-4}$ and $1.25 \cdot 10^{-1}$ for signaling volume, low-bandwidth channel use and energy consumption respectively. This static assignment of weights was proposed for evaluation purposes. Tuning the weights lies outside the scope of this paper; it will be dealt with at the optimization stage of MILCO's design.

TABLE VIII

QUANTIFICATION FOR THE FACTORS USED IN THE COST FUNCTION

\begin{tabular}{|c|c|c|c|}
\hline Technique & $\begin{array}{r}\text { Signaling volume } \\
\text { (bits) }\end{array}$ & $\begin{array}{r}\text { Low-bandwidth channel } \\
\text { use (ns) }\end{array}$ & $\begin{array}{r}\text { Energy } \\
\text { consumption }\end{array}$ \\
\hline Cell-ID & 0 & 0 & 0 \\
\hline $\begin{array}{l}\text { OTDOA } \\
\text { (AS) }\end{array}$ & $375+134 \cdot N_{N B}$ & \multirow{5}{*}[\frac{2\cdot10^{9}}{155\mathrm{Mbps}}+\frac{2\cdot10^{9}}{384\mathrm{Kbps}}]{} & $N_{N B}$ \\
\hline $\begin{array}{l}\text { OTDOA } \\
\text { (NAS) }\end{array}$ & 268 & & $N_{N B}$ \\
\hline A-GPS (AS) & $473+1199 \cdot N_{S A T}$ & & $N_{S A T}$ \\
\hline $\begin{array}{l}\text { A-GPS } \\
\text { (NAS) }\end{array}$ & $461+647 \cdot N_{S A T}$ & & $N_{S A T}$ \\
\hline Hybrid & $\begin{array}{r}653+134 \cdot N_{N B}+ \\
1254 \cdot N_{S A T}\end{array}$ & & $N_{N B}+N_{S A T}$ \\
\hline
\end{tabular}

Position requests were generated by a single service, and the accuracy was uniformly distributed from 10 meters (e.g. tracing, tracking and emergency services, etc.) to 2 kilometers (e.g. location-based information, enhanced call routing, etc.). 
The response time required was also uniformly distributed between 0 seconds, which means that the location must be provided immediately (e.g. emergency services), and 60 seconds (e.g. push services). Both accuracy and delay constrain the QoS, i.e. not satisfying one of the constraints results in QoS failure (3GPP allows other QoS approaches but the most restrictive one was used in this study).

\section{IV.Performance ANALysis}

This section analyzes the performance of MILCO and focuses on the resources used by each location-based service and the location traffic being carried. Location techniques used as standalone are included for comparison.

Fig. 4 shows the percentage of unsuccessful LBS in a scenario loaded with 30 requests per second when only the cost function was used (i.e. the location cache and concurrence manager were disabled). The failures of the mobile-based location techniques due to handoffs were not taken into account. The best results for the standalone location techniques were obtained by A-GPS, with a $17.72 \%$ LBS failure rate. Using MILCO reduced this figure by $57.85 \%$ and better results were obtained in comparison with the other techniques. The results achieved by exclusively using the OTDOA and A-GPS were similar. This is because accuracy and response time constrain the QoS in both cases. Thus, response time constrains the A-GPS results and accuracy does the same for the OTDOA. The hybrid technique was also constrained by the long response times, resulting in a low rate of success for LBS.

Performance was expected to be improved by the input modules. Hereafter, all the measurements were taken by implementing all the modules (i.e the location cache and concurrence manager were also enabled). Fig. 5 plots the evolution of the unsuccessful LBS requests with the load, both taking into account and disregarding the mobility of the terminal (i.e. LBS failures due to handoff). The inter-arrival time stands for the time that elapses between two consecutive location requests, i.e. the shorter the inter-arrival time, the heavier the load. As shown, the unsuccessful LBS rate was higher when mobility was accounted for. This is because handoffs interrupt all ongoing requests, regardless of the handoff result [12]. The results for which mobility was taken into account and disregarding are similar, since the average speed of the mobile station was very low. However, greater differences were expected with higher average MS speeds.

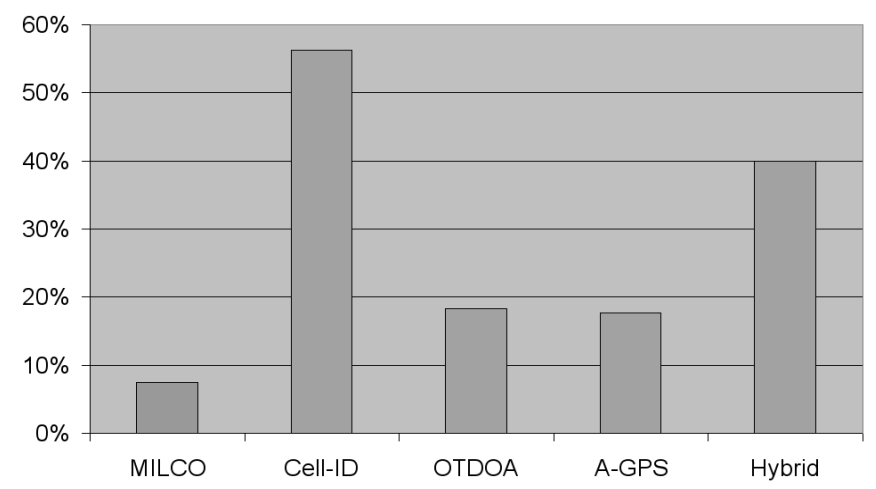

Fig. 4. Percentage of unsuccessful location processes

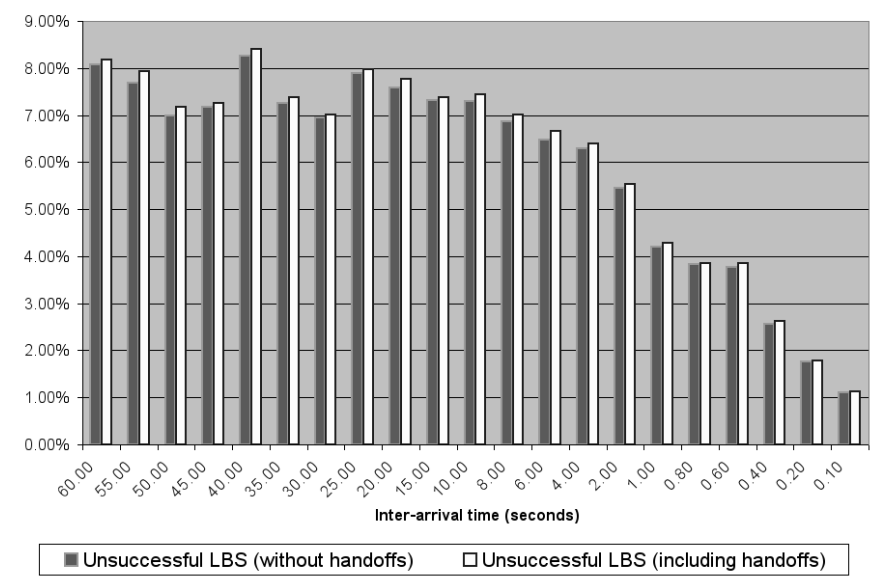

Fig. 5. Evolution of unsuccessful LBS with MILCO

Fig. 5 shows that at medium/low request rates (i.e. inter-arrival times longer than 10 seconds), MILCO achieved an LCS failure of between 7 and $8 \%$. However, the higher the load, the lower the LCS failure rate, since less time is spent between consecutive location requests; therefore, the cache and concurrence features are more likely to be used. This proves that the scalability of the proposed approach is guaranteed: for heavier loads the input modules' performance reduces the percentage of unsuccessful requests.

Reducing the use of resources is another advantage with MILCO. Fig. 6 plots the average and standard deviation of the number of location techniques run per location process. This figure shows that at lighter loads (i.e. inter-arrival times longer than 20 seconds), the average number of location techniques remained very close to 1 . This was because, at these rates, concurrence and cache were not useful and only the cost function was used. Therefore, few processes had enough time to run more than one location technique (if necessary) and fulfill the response-time requirements. The number of location techniques that were being used fell as the load increased, since input modules handle more requests: requests handled by input modules do not involve running any techniques. In addition, standard deviation of this variable increases with traffic for rates greater than 1 request per second. This is due to the difference between requests served by the cost function (which involves at least 1 location technique) and input modules (in which no location techniques are run). With reference to the top right-hand corner of Fig. 6, for interarrival times of less than 1 second the standard deviation fell, since the number of location techniques being served by the location cache and concurrence manager was much greater than the number dealt with by the cost function, reaching $88 \%$.

Fig. 7 shows the average resources consumed by MILCO according to the definition provided in (2) and the percentage of LBS that made use of the location cache and concurrent LCS. In this figure, the percentage of resource use is provided relative to the hybrid technique (considered to be $100 \%$ ), according to the data in Table VIII and the weights used in the cost function. Therefore, average resource use represents the cost of requests handled by MILCO with reference to the consumption of resources that is achieved when only the hybrid technique was used. In lightly loaded scenarios, the location cache and concurrence manager were seldom used. Fig. 7 shows that fewer than 2 and 5\% of requests were 


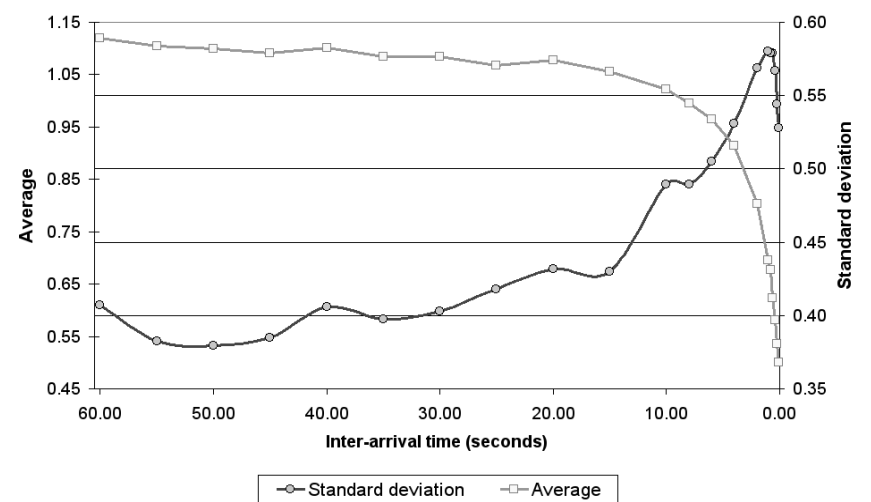

Fig. 6. Average number of techniques run per location process

handled successfully by the location cache and concurrence manager in these scenarios. This means that MILCO was reduced to the cost function. However, using this feature only, reduced the use of resources by more than $70 \%$, since the location techniques executed were selected according to the QoS requested, the features of the network and the capabilities of the base station. Better figures were achieved when the load increased, reaching a saving of up to $95 \%$. This improvement was due to the increasing use of the location cache and concurrence manager, since these modules deal with location requests at no cost. Fig. 7 shows that in the case of heavier loads, most of the requests were handled by the location cache or concurrence manager. Indeed, in the heaviest load scenario (10 requests per second), $88 \%$ of the LBS were successfully handled by the input modules.

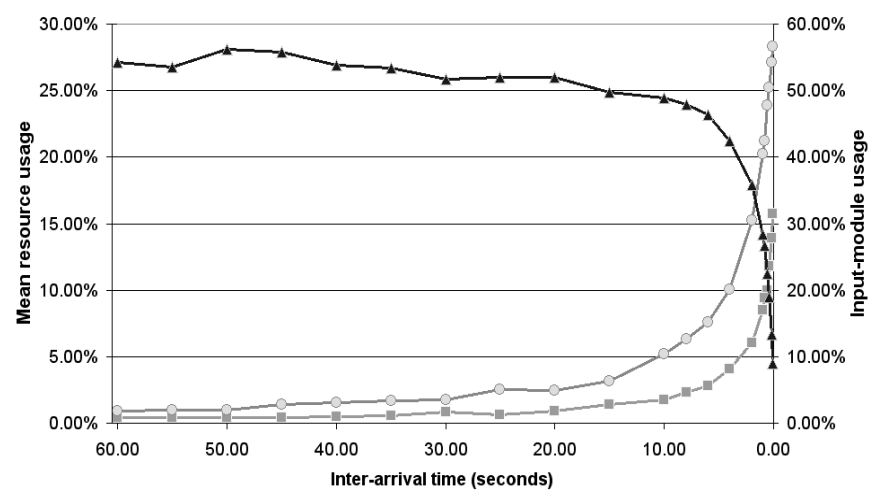

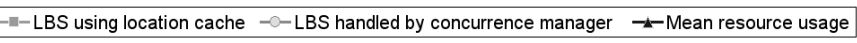

Fig. 7. Average resource use with MILCO

Even though the location concurrence algorithm provided several advantages in terms of the load carried and a reduction in network resource use, its performance was far from optimal. Fig. 8 shows the percentage of unsuccessful LBS (not including those caused by handoff processes) and the percentage of these unsuccessful LBS for which concurrence was used. Simulations show that most LCS failures at higher loads were due to the concurrence manager (e.g. at 10 requests per second, the concurrence manager was involved in the $72.58 \%$ of the LCS failures). However, this is not particularly relevant, as the percentage of unsuccessful LCS at these rates was very low.

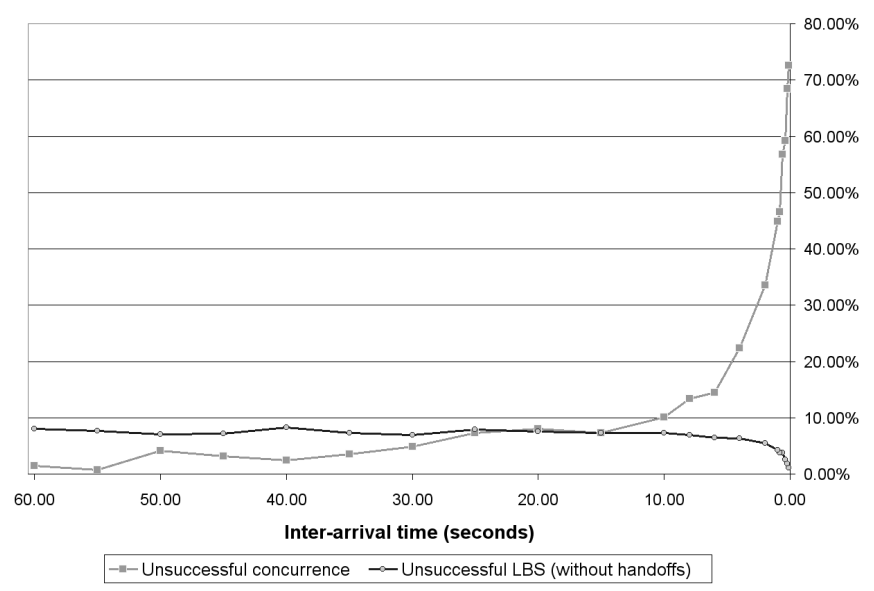

Fig. 8. Percentage of unsuccessful LBS and concurrence misses

\section{Conclusion}

This paper presents MILCO, a middleware that aims to reduce the use of network resources and maximize the traffic carried by location platforms. MILCO is composed of several modules, three of which are particularly important: the cost function, the location cache and the concurrence manager. Just by using the cost function, MILCO reduces network resource use (defined by several factors) by more than $70 \%$ in comparison with systems that exclusively implement a hybrid A-GPS/OTDOA technology. Significant savings are also achieved in comparison with other standalone techniques. Therefore, MILCO improves system performance just by selecting the technique most suited to the QoS requested. When all the modules are used together, this saving goes up to $95 \%$. This means that more traffic can be carried using the same amount of network resources. The results also show that MILCO reduces the percentage of unsuccessful location requests by $57.85 \%$ in comparison with A-GPS, which is the technique that achieves the best results when used as standalone. Better results are obtained when the middleware is compared with other techniques. The location cache and concurrence manager mean that MILCO is a scalable system, since increasing the load reduces the unsuccessful LBS rate and resource use.

\section{REFERENCES}

[1] S.S. Soliman, C.E. Wheatley: Geolocation Technologies and Applications for Third Generation Wireless, Wireless Communications and Mobile Computing, No.2, pp. 229-251, May 2002.

[2] A. Küpper: Location-Based Services: Fundamentals and Operation, John Wiley \& Sons, Aug. 2005.

[3] F. Barceló, I. Martin-Escalona: Coverage of Hybrid TerrestrialSatellite Location in Mobile Communications, Fifth European Wireless Conference: Mobile and Wireless Systems beyond 3G, pp. 475-479, Sept. 2004.

[4] S. Venkatraman, J.Jr. Caffery: Hybrid TOA/AOA techniques for mobile location in non-line-of-sight environments, IEEE Wireless Communications and Networking Conference (WCNC 2004), vol. 1, pp. 274-278, March 2004. 
[5] H.C. Son, J.G. Lee, G.I. Jee: Mobile station location using hybrid GPS and a wireless network, IEEE Vehicular Technology Conference (VTC 2003-Spring), vol. 4, pp. 2716 -2720, April 2003.

[6] S. Soliman, P. Agashe, I. Fernandez, A. Vayanos, P. Gaal, M. Oljaca: gpsOneTM: a hybrid position location system, IEEE Spread Spectrum Techniques and Applications, vol. 1, pp. 330335, Sept. 2000.

[7] S. Rooney, P. Chippendale, R. Choony, C. Le Roux, B. Honary: Accurate vehicular positioning using a DAB-GSM hybrid system, IEEE Vehicular Technology Conference (VTC 2000Spring), vol. 1, pp. 97-101, May 2000.

[8] I. Martin-Escalona, F. Barcelo: Optimization of the Cost of Providing Location Services in Mobile Cellular Networks, 15th IEEE International Symposium on Personal, Indoor and Mobile Radio Communications (PIMRC 2004), Vol. 3, pp. 2076-2081, Sept. 2004.

[9] M. Spanoudakis, A. Batistakis, I. Priggouris, A. Ioannidis, S. Hadjiefthymiades, L. Merakos: Extensible platform for location based services provisioning, 4th International Conference on Web Information Systems Engineering Workshops. 72- 79, Dec. 2003.

[10] Y. Chen, W. Lu, X. Chen, L. Tang, F. Rao, Q. Wang, L. Zhang: Location aware messaging - integrating LBS middleware and converged services, IEEE International Conference on eBusiness Engineering 2005 (ICEBE 2005), pp. 419-426, Oct. 2005.

[11] U. Ahmad, U. Nasir, M. Iqbal, Y.K. Lee, S.Y. Lee, I. Hwang: On building a reflective middleware service for locationawareness, IEEE International conference on Embedded and Real-Time Computing Systems and Applications 2005, pp. 429442, August 2005.

[12] 3GPP TS 23.271: Functional Stage 2 Description of Location Services (LCS).R6, 2004.

[13] P. Biswas, S. Han, J. Wu: Location Caching in the Mobile Middleware Platform, 3rd International Conference on Mobile Data Management, pp. 172-174, Jan. 2002.

[14] J. Zander, S.L. Kim: Radio Resource Management for Wireless Networks, Artech House, 2001.

[15] A. Capone, S. Redana: Call Admission Control Techniques for UMTS, IEEE VTS 54th Vehicular Technology Conference, 2001. VTC 2001 Fall, Vol. 2 , pp. 925-929, Oct. 2001.

[16] L. Nuyami, X. Lagrange, P. Godlewski: A Power Control Algorithm for $3 G$ WCDMA System, European Wireless 2002, Feb. 2002.

[17] H. Holma, A. Toskala: WCDMA for UMTS, John Wiley \& Sons, 2000.

[18] ETSI TR 125.942: RF system scenarios, 2004.

[19] EMILY.IST-2000-26040: EMILY system trials report, 2005.

[20] G. Wilde: Why are LB\$ a Long Time Coming?, Consulting paper by BWCS, 2002.

[21] J.Y. Lee, W.C..Y. Lee: Optimize CDMA System Capacity with Location, Proc. of IEEE PIMRC 2001, pp. 17-21, Oct. 2001.
[22] S.S. Wang, M. Green, M. Malkawi: Mobile Positioning Technologies and Location Services, IEEE Radio and Wireless Conference, pp. 9-12, Aug. 2002.

[23] W.S. Soh, H.S. Kim: A predictive Bandwidth Reservation Using Mobile Positioning and Road Topology Information, IEEE Transactions on Networking, Vol. 15, Issue 5, pp. 1078-1091, Oct. 2006.

[24] S. Goebbels, M. Siebert, M., Schinnenburg, M. Lott: Simulative evaluation of location-aided handover in wireless heterogeneous systems, 15th Personal, Indoor and Mobile Radio Communications (PIMRC 2004), Vol. 2, pp. 1080-1084, Sept. 2004.

[25] C. Ming-Hsing, M.A. Bassiouni: Predictive schemes for handoff priorization in cellular networks based on mobile positioning, IEEE Journal on Selected Areas in Communications, Vol. 18, Issue 3, pp. 510-522, March 2000.

[26] 3GPP TS 25.331: Radio Resource Control (RRC) protocol specification, 2005.

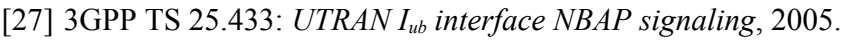

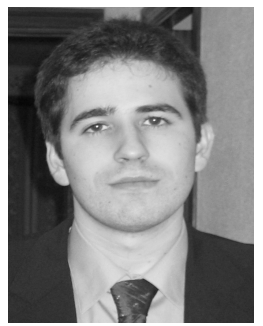

Israel Martin-Escalona is a lecturer at the Department of Telematics Engineering of the Technical University of Catalonia (UPC). He earned a degree in Telecommunications Engineering in 2001 and is currently applying for the degree in Computer Science at UPC. He began his $\mathrm{PhD}$ in Telematics Engineering in 2002 at UPC. Since then, his research has focused on mobile networks, particularly the performance of location systems in cellular environments. He is currently involved in several research projects funded by the Spanish government and the European Commission.

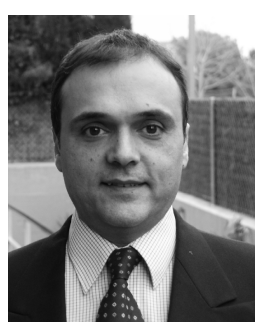

Francisco Barcelo earned a degree in Telecommunications Engineering and a $\mathrm{PhD}$ from the Technical University of Catalonia (UPC) in 1986 and 1997, respectively. In 1987 he joined UPC's School of Telecommunications Engineering of Barcelona, where he has been teaching communication network design and planning. Having graduated, he carried out research on digital network synchronization and switching. Since 1997 he has been an associate professor at the Department of Telematic Engineering at UPC. He also acts as a consultant in the telecommunications industry in Spain and is currently involved in several research projects funded by the Spanish government and the European Commission. His current research interests lie in studying the evaluation of the capacity and performance of wireless networks: teletraffic analysis of wireless networks, traffic modeling, resource assignment and third generation protocols. 